\title{
The Vacuum-Assisted System as A Treatment of Deep Sternal Wound Infections Following Cardiac Surgery. Cairo University Hospitals Experience Ahmed S Mahmoud ${ }^{1 *}$, Rafik F B Soliman' ${ }^{2}$, Fouad M Rasekh ${ }^{1}$
}

${ }^{1}$ Department of Cardiothoracic Surgery, Faculty of Medicine, Cairo University, ${ }^{2}$ Department of Cardiothoracic Surgery, Faculty of Medicine, Menofia University

"Corresponding authors: Ahmed Sayed Hussein, Mobile: (+20) 01090991111, E-mail: drahmed 755@ gmail.com

\begin{abstract}
Background: The vacuum-assisted system in wound closure (VAC) is a new modality, which is recently used widely as a treatment of severely infected wounds. It is a noninvasive way that improves healing of severely infected wounds that fail to respond to conventional ways of treatment. This type of treatment depends on applying air tight controlled negative pressure suction directly to the infected wounds. The mechanism of action of this treatment depends on allowing the growth of healthy granulation tissues of the infected wounds.

Objective: To evaluate the effect of vacuum assisted suction in treatment of deep sternal wound infection after cardiac surgery.

Patients and Methods: In Cairo University Hospitals, in the period between January 2018 and January 2019, 17 patients (6 men, 11 women) with a median age of 62.5 years (range 50 to 75 years) underwent open heart surgery, which was complicated by sternal dehiscence and deep sternal wound infection. All those patients were subjected to the VAC system before any surgical intervention.

Results: All patients involved in this study had complete wound healing without need of any major surgical intervention (muscle or omental flaps) even without wound debridement. The VAC system was applied for a mean of 21.5 days (range 15 to 28 days), VAC system was removed when the wound became completely clean with healthy granulation tissues. After removal of the VAC system, 9 patients $(52.9 \%)$ were subjected to closure of the skin and subcutaneous tissues by interrupted stitches while the remaining 8 patients $(47.1 \%)$ the wound was completely closed in all layers up to the skin. Duration of hospital stay varied from 22 to 45 days (median 33.5 days). There were no mortalities among all the patients who were involved in this study.
\end{abstract}

Conclusion: The VAC system is a noninvasive, safe and very effective as a treatment for deep sternal wound infections following cardiac surgery.

Keywords: Deep sternal wound infections, Vacuum assisted system.

\section{INTRODUCTION}

Deep sternal wound infections after heart surgery are a major complication and sometimes a lifethreatening condition, despite significant improvements in wound care and antibiotic medications, the incidence of these complications is around $4 \%$ in the best cardiac centers with a mortality rate around $15 \%(\mathbf{1}, \mathbf{2})$.

Over the past decades, cardiac surgeons have tried numerous methods of treating deep sternal wound infections after heart surgery, ranging from topical lavage and wound washing with antibiotics to aggressive surgical interventions such as reconstructive surgery with pectoral flaps or omental flaps ${ }^{(3)}$. The idea of VAC was first used in 1996 in the treatment of pressure ulcers in a bedridden patient with excellent results ${ }^{(4)}$, and since then VAC has become the cornerstone of treating highly contagious wounds $(3,4)$. The mechanism of action of the VAC is based on the expansion of the microcirculation by controlled negative pressure on the affected wound, which promotes healthy granulation tissue proliferation ${ }^{(4)}$.

Aim of present work was to evaluate the effect of vacuum assisted suction in treatment of deep sternal wound infection after cardiac surgery.
In Cairo University Hospitals, in the period between January 2018 and January 2019, 17 patients (6 men, 11 and women) with a median age of 62.5 years (range 50 to 75 years) underwent open heart surgery and complicated by sternal dehiscence and deep sternal wound infection, all those patients were subjected to the VAC system. All infected patients underwent coronary artery bypass surgery $(\mathrm{CABG})$ with use the left internal thoracic artery, the left radial artery and the greater saphenous vein and no bilateral internal mammary artery were used. The preoperative patient's characteristics and risk factors were reported in table (1).

Table (1): Patient's characteristics and risk factors.

\begin{tabular}{|l|c|}
\hline Age (years) & $50-75$ mean (62.5) \\
\hline Female patients & $11(64.7 \%)$ \\
\hline Diabetes & $6(35.2 \%)$ \\
\hline Body mass index> 35 & $4(23.5 \%)$ \\
\hline $\begin{array}{l}\text { Chronic obstructive } \\
\text { pulmonary disease }\end{array}$ & $3(17.6 \%)$ \\
\hline $\begin{array}{l}\text { Prolonged postoperative } \\
\text { mechanical ventilation }\end{array}$ & $2(11.8 \%)$ \\
\hline Redo surgery & $1(5.9 \%)$ \\
\hline Postoperative reopening & $1(5.9 \%)$ \\
\hline
\end{tabular}

\section{PATIENTS AND METHODS}

This article is an open access article distributed under the terms and conditions of the Creative Commons Attribution (CC BY-SA) license (http://creativecommons.org/licenses/by/4.0/) 
Patient was diagnosed as postoperative deep sternal wound infection with the following: discharge of purulent fluid or pus from sternal wound, instability of the sternum or dehiscence, fever, pain and tenderness in the sternal wound, redness and hotness in the sternal wound, leukocytosis with shift to the left, and/or computerized tomography (C.T) of chest showed separation between the two edges of the sternum.

Patients wounds were followed up for deep sternal wound infections postoperatively and time between the primary open heart surgery and diagnosis of deep sternal wound infection according to the previous parameters varied from 5 to 10 days with average 7.5 days. Among the 17 patients there were 2 (11.8\%) patients discharged from hospital and came back to the outpatient clinic with wound discharge and readmitted again to the hospital.

In the all 17 patients diagnosed as postoperative deep sternal wound infection; the VAC system were applied. In 10 patients $(58.8 \%)$ the skin was closed so as a small opening ranged from 2 to $3 \mathrm{~cm}$ in the skin was done to insert the VAC, while in remaining 7 patients $(41.2 \%)$ the wound was opened, so debridement of infected tissues was done then VAC system was applied to the wound with contours suction $150 \mathrm{~mm} \mathrm{Hg}$. In all cases proper wash with saline and irrigation with diluted iodine of the wound was done before insertion of the VAC. In all patients a swab from wound discharge was obtained and sent to the laboratory for culture and sensitivity to detect the bacterial organism to give intravenous antibiotic according to the results. Meanwhile, all patients received intravenous broad spectrum antibiotics covering gram positive and gram negative bacilli until we get the results of the culture and sensitivity. The whole procedure was done in the department without need of operating theatre.

In all patients with VAC, every three days the VAC system was changed and a new dressing, adhesive plaster and connecting tube was inserted, wound every time was checked for growth of healthy granulation tissues and wound discharge and swab from wound discharge was obtained every 6 days to be sent to the laboratory for culture and sensitivity to shift to another antibiotics accordingly.

\section{Ethical approval}

An approval of the study was obtained from Cairo University academic and ethical committee. Every patient signed an informed written consent for acceptance of the operation.

\section{Statistical methods}

Recorded data were analyzed using the statistical package for social sciences, version 20.0 (SPSS Inc., Chicago, Illinois, USA). Quantitative data were expressed as mean \pm standard deviation (SD). Qualitative data were expressed as frequency and percentage.

\section{RESULTS}

In the all 17 patients were involved in this study complete sternal wound healing was achieved without any further reconstructive surgical intervention and sternal stability was restored in all patients. VAC system was a definite treatment for all patients.

The post VAC data are shown in table 2. No intensive care unit (ICU) stay was needed as all patients were treated in the department. All patients during the hospital stay received broad spectrum intravenous antibiotics, which was changed according to the results of culture of the wound swab and after discharge from hospital all patients received oral antibiotics according to the result of the last culture of wound swab for 10 days. VAC was removed with growth of healthy granulation tissues, sternum became stable and local improvement of signs of infection. No morbidity or mortality was reported among the all 17 patients and all patients were followed up for 10 days after discharge from hospital with excellent results. There was no mortality among all patients treated with VAC.

In 10 patients (58.8\%) the wound was completely closed including skin and subcutaneous tissues while in 7 patients $(41.2 \%)$ the wound was opened after removal of VAC and it was closed in one layer with secondary interrupted prolene stitches with subcutaneous injection of lidocaine $2 \%$ at site of stiches and procedure was done in the department without need of operating theatre.

Bacterial cultures isolated Staphylococcus aureus in 7 patients (41.2\%), Pseudomonas aeruginosa in 5 patients $(29.4 \%)$ and Klebsiella pneumoniae in 5 patients $(29.4 \%)$.

Table (2): Post VAC data.

\begin{tabular}{|l|l|}
\hline Post vacuum data & \\
\hline $\begin{array}{l}\text { Time between primary } \\
\text { surgery and VAC insertion }\end{array}$ & 5-10 days (mean 7.5) \\
\hline $\begin{array}{l}\text { Need of skin opening for } \\
\text { VAC }\end{array}$ & 10 patients (58.8\%) \\
\hline $\begin{array}{l}\text { Time from insertion to } \\
\text { removal of VAC }\end{array}$ & $\begin{array}{l}15-28 \text { days } \\
\text { (mean12.5) }\end{array}$ \\
\hline Need of operating theatre & None \\
\hline ICU stay & None \\
\hline Total hospital stay & $22-45$ days (mean 33.5) \\
\hline
\end{tabular}

\section{Discussion:}

The idea of VAC was first time used by Argenta and Morykwas in 1996, and since that time the VAC became an important tool in treatment of severely infected time as it gave very good results without surgical intervention ${ }^{(4)}$. Before use of VAC, the treatment of severely infected wounds was mainly by 
aggressive debridement and keep the wound opened to allow free drainage of any discharge and this way had a reasonable results in most of wound infections but with severe wound infections, as deep sternal wound infections, it was not so ${ }^{(3,4)}$. VAC system has many advantages over the conventional treatment of wound infections: It allows continues suction of fluids and exudate thus reducing edema of infected tissues, it decreases the bacterial growth in the infected wounds, and the most important advantage of exerting negative pressure suction to the infected wound is to encourage dilatation of microvascular circulation which increases blood supply and enhances proliferation and growth of healthy granulation tissues ${ }^{(5,6)}$.

In our study there was no ICU stay and no need for operating theatre as the whole procedure can be done completely in the department compared with other options as reconstructive surgery; whether with pectoral or omental flap, which is done in operating theatre and with general anesthesia and needs stay in the ICU and definitely longer hospital stay with more pain and needs aggressive analgesics like opioids.

Timing of insertion of VAC in deep sternal wound infections after cardiac surgery is very important as the earlier we apply it is the better the results. If we compare our results by study done by Fleck et al. ${ }^{(7)}$, they had the same study over 11 patients and 6 of them $(54.5 \%)$ underwent reconstructive pectoral flap after VAC with ICU stay, the mean time of insertion of VAC was 9.1 days while in our study all patient healed by VAC without further reconstructive surgery; the mean time of insertion of VAC was 7.5 days. So it is very important to apply VAC once deep sternal wound infection after cardiac surgery is diagnosed.

An important factor in treatment of deep sternal wound infection after cardiac surgery by VAC was observed is sternal stability, which was regained in all patients, which is very important to allow normal respiratory movement instead of paradoxical movements and also it avoids cardiac injuries, which may occur after pectoral or muscle flaps by sternal edges ${ }^{(8-10)}$.

There is an important advantage of VAC over reconstructive flap as patients can be treated at home with VAC as it is easy to deal with and patient can go to outpatient clinic every 3 days to change the VAC, which is not an option in omental or pectoral flap, thus we can significantly shorten hospital stay $(\mathbf{9 , 1 1}, \mathbf{1 2})$.
The main limiting factor in our study was the few number of cases involved in this study.

\section{CONCLUSION}

From results of our study we conclude that VAC is noninvasive, safe, reliable and very efficient in treatment of deep sternal wound infection after cardiac surgery.

\section{REFERENCES}

1. Loop F, Lytle B, Cosgrove D et al. (1990): Sternal wound complications after isolated coronary artery bypass grafting. Ann Thorac Surg., 49: 179-187

2. De Feo M, Renzulli A, Ismeno G et al. (2001): Variables predicting adverse outcome in patients with deep sternal wound infection. Ann Thorac Surg., 71: 324-331.

3. Francel T, Kouchoukos N (2001): A rational approach to wound difficulties after sternotomy. Ann Thorac Surg., 72: 1419-1429

4. Shumacker H, Mandelbaum I (1963): Continuous antibiotic irrigation in the treatment of infection. Arch Surg., 86: 384-387

5. Jurkiewicz M, Bostwick J, Hester T et al. (1980): Infected median sternotomy wound. Ann Surg., 191: 738-744

6. Rand R, Cochran R, Aziz S et al. (1998): Prospective trial of catheter irrigation and muscle flaps for sternal wound infection. Ann Thorac Surg., 65: 1046-1049.

7. Fleck T, Fleck M, Moidl R et al. (2002): The vacum assisted closure system for treatment of deep sternal wound infections after cardiac surgery, The Ann Thoracic Surg., 74: 1596-1600.

8. Borer A, Gilad J, Meydan N et al. (2001): Impact of active monitoring of infection control practices on deep sternal infection after open heart surgery. Ann Thorac Surg., 72: 515-520.

9. Yasuura K, Okamoto H, Morita S et al. (1998): Results of omental flap transposition for deep sternal wound infection after cardiovascular surgery. Ann Surg., 227: 455-459.

10.Jones G, Jurkiewicz M, Bostwick J et al. (1997): Management of the infected sternotomy wound with muscle flaps. Ann Surg., 225: 766-768.

11.El Gammel M, Yonan N, Hassan $R$ et al. (1998): Treatment of mediastinitis. Ann Thorac Surg., 65: 4147.

12. Morykwas M, Argenta L, Shelton-Brown E et al. (1997): Vacuum assisted closure. Ann Plast Surg., 38: 553-562. 